\title{
Gas-Phase Nitroaromatic Detection Based on Sulfonated Tetrafluoroethylene and Its Field Application
}

\author{
Sungjun Lee and Junghoon Lee* \\ Seoul National University, 1 Gwanak-ro, Gwanak-gu, Seoul 151-744, South Korea
}

(Received July 26, 2014; accepted October 9, 2014)

Key words: nitroaromatic, explosive, sensor, sulfonated tetrafluoroethylene, Nafion ${ }^{\circledR}$, quartz crystal microbalance

We report on the use of sulfonated tetrafluoroethylene (ST) for explosive sensing and its feasibility for field application. The sensing performance of ST is verified with a quartz crystal microbalance (QCM) setup and a certified standard gas generator. ST can directly capture 2,4-dinitrotoluene (DNT), an indicator molecule of explosives, from the gas. Unlike other newly developed technologies for explosive detection, the use of ST does not need a humid or aqueous environment, which is difficult to maintain in field application. A handheld explosive detector is developed on the basis of ST and a miniaturized QCM system. Its sensitivity is $-33.52 \mathrm{~Hz} / \mathrm{ppb}$ and its limit of detection (LOD) is $1.19 \mathrm{ppb}$. Furthermore, a semifield test is performed with the device and a field-reproducing setup. This work successively demonstrates the explosive detection capability in an uncontrolled field environment.

\section{Introduction}

The detection of hidden explosives, such as landmines and unexploded ordnance (UXO), and their removal are key areas for maintaining a quality life on a global scale. About 4000 victims were reported by the "Landmine and Cluster Munition Monitor", a humanitarian nongovernmental organization (NGO), in 2012.(1) Civilian casualties reached up to $78 \%$ and children were $47 \%$ of the victims. This field requires an immediate response of technology to address the issues of detection and removal of such life-threatening chemicals and explosives.

Despite the gravity of the problems as outlined above, detection methods for hidden explosives still remain on a primitive level. A metal detection method and a groundpenetrating radar, which were developed more than 50 years ago, are still considered as

*Corresponding author: e-mail: jleenano@snu.ac.kr 
the most practical approaches used in the field, despite their poor detection reliability. Since they rely on an indirect detection scheme to sense the housing material rather than the explosive element itself, their false alarm rates are significant.

A direct detection method needs to be developed to solve this issue fundamentally. However, the development process is challenging owing to the lack of an explosivesensing material compatible with field application. ${ }^{(2)}$ Much effort has been made to develop reliable direct explosive detection methods, such as bioreceptor-based sensing, ${ }^{(3,4)}$ synthetic-material-based sensing, ${ }^{(5-8)}$ and Meisenheimer-complex-based sensing. ${ }^{(9)}$ However, the bioreceptor and the Meisenheimer complex approaches have a sensitivity issue under a dry condition. To maintain their activity, a wet environment is needed, which is difficult to maintain in a field environment. The synthetic sensing material has a poor selectivity issue. To the best of our knowledge, a field-applicable approach has not been fully developed yet.

The use of sulfonated tetrafluoroethylene (ST, Nafion ${ }^{\circledR}$ ) can solve these problems. The sulfone functional group in ST can be used to form the Meisenheimer complex and ST does not need a wet environment for the reaction. The detailed mechanism will be described.

Here, we demonstrate a "gas-phase" 2,4-dinitrotoluene (DNT) detection using ST as a DNT-sensing material and its field application. The sensing performance of ST is verified with a commercial quartz crystal microbalance (QCM) setup and a standard DNT gas generator. High selectivity is confirmed against toluene. We also developed a handheld explosive detector based on ST. It shows $-33.52 \mathrm{~Hz} / \mathrm{ppb}$ sensitivity and a ppblevel limit of detection (LOD) without any complicated assistance device for humidity control. The portable device also demonstrates field application feasibility with the help of a field-reproducing setup.

\section{Materials and Methods}

\subsection{Test setup}

The interaction between ST and a nitroaromatic (here, DNT, an indicator of nitroaromatic explosives) was examined using a commercial QCM system (QCM100, SRS, USA) and a standard gas generator (BSG-100S, Bon Gil Inc., Korea) (Fig. 1). The QCM had a $\mathrm{Au} / \mathrm{Cr}$-coated circular (diameter, 1 inch) quartz crystal with $5 \mathrm{MHz}$ resonance frequency. The quartz qrystal was assembled in a Teflon ${ }^{\circledR}$ fluidic cell and directly connected to the standard gas generator to prevent sample contamination. The standard gas generator had a certified permeation tube (Certified from KIN-TEK Inc., USA) and the generator could produce DNT vapor from 1 to $100 \mathrm{ppb}$. Compressed highpurity nitrogen with a typical flow rate of $5 \mathrm{slm}$ was used as a carrier gas. The change in QCM frequency was monitored using an HP 53151A frequency counter (USA) and a general purpose interface bus (GPIB)-linked data acquisition computer through LabView 2013 (National Instrument, USA) as an interfacing software program.

To test the affinity of ST to the DNT, the quartz crystal was coated with ST. Prior to the ST coating, the quartz crystal was cleaned with a piranha solution $\left(\mathrm{H}_{2} \mathrm{SO}_{4}: \mathrm{H}_{2} \mathrm{O}_{2}\right.$ $=4: 1$ solution) for 10 min followed by deionized (DI) water rinsing and $\mathrm{N}_{2}$ blowing for 


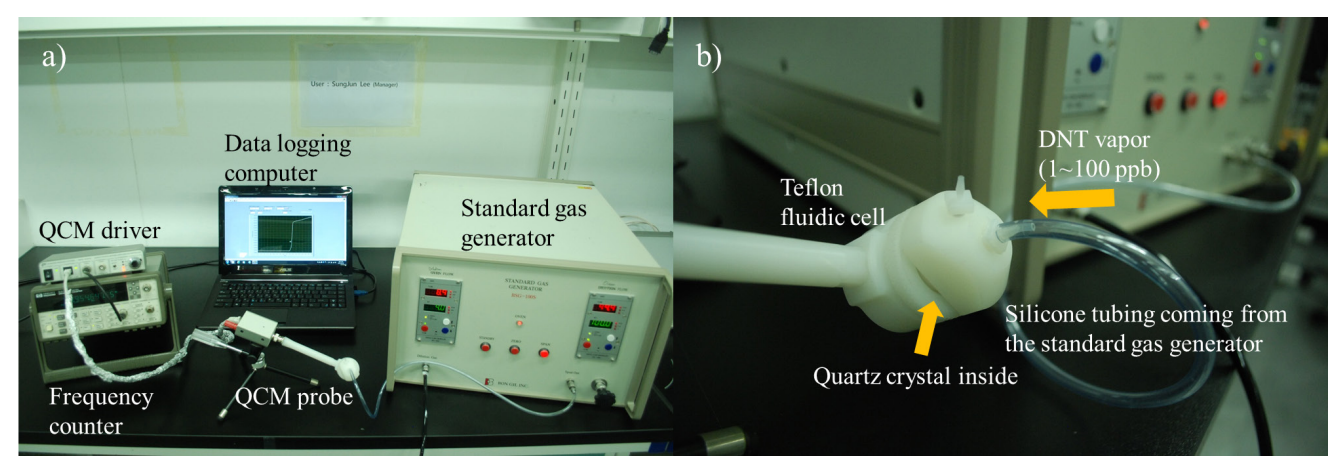

Fig. 1. (Color online) Experimental setup. (a) A certified standard gas generator (BSG-100S, Bon Gil Inc., Korea. Certified from KIN-TEK Inc., USA) and a commercial QCM (QCM-100, SRS, USA) were employed for reliable verification. (b) A teflon fluidic cell and a tubing directly linked to the standard gas generator were used to prevent vapor contamination.

dehydration. For coating, the ST solution $\left(20 \mathrm{wt} \%\right.$ Nafion ${ }^{\circledR}$ perfluorinated resin solution, Sigma-Aldrich, USA) was diluted 10 times with ethanol and $20 \mu 1$ of the diluted solution was dropped on the quartz crystal. The crystal was dried for $5 \mathrm{~h}$ at room temperature, which was followed by overnight drying at $70{ }^{\circ} \mathrm{C}$ in a conventional oven. The typical membrane coating thickness was measured as $20 \mu \mathrm{m}$ using the scratch method. The prepared sample was stored in a sealed container before use.

\subsection{Control experiment setup}

A control experiment was performed using a vapor chamber of $200 \times 300 \times 1000$ $\mathrm{mm}^{3}$ size (about $150 \mathrm{~L}$ in volume). A QCM probe was fixed on a tripod and placed at the bottom of the chamber. The test gas was produced by natural evaporation. A cotton with toluene solution was placed at the corner of the chamber after the stabilization of the QCM. The frequency response to the toluene was measured during its vaporization. The gas concentration in the chamber was monitored with a commercial gas sensor (Series 500 Handheld Monitor, Aeroqual Co., New Zealand). The high-precision commercially available sensor was placed near the QCM probe to measure the toluene toluene concentration in air during the experiment.

\subsection{Handheld DNT detector}

A handheld DNT detector based on a miniaturized QCM and ST was developed (Fig. 2). The QCM contained a simple and robust positive-feedback circuit and a quartz crystal oscillator. An oscillating circuit was configured using inverters (HEF4069UBP, NXP Co., Netherlands). The frequency was measured using a serially linked 12-stage counter (HEF4040BT, Philips Co., Netherlands). The measured binary frequencies were displayed on an LCD screen and transmitted to a data acquisition computer via a Bluetooth link. 


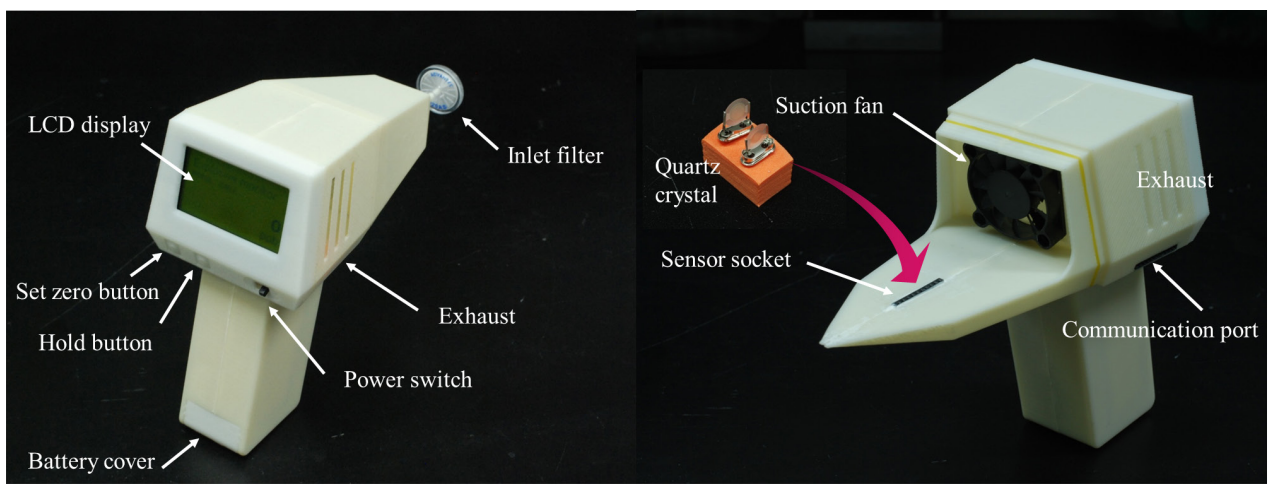

Fig. 2. (Color online) Developed handheld device. The device could be operated as a stand-alone device without any need for external equipment.

\subsection{Handheld device test setup}

The developed handheld device was examined with a bottle containing high-purity DNT (Fig. 3) and a field-reproducing setup (Fig. 4). Environmental factors, such as temperature, humidity, and wind, were not controlled in these experiments for substantial field reproduction.

The DNT bottle (2,4-dinitrotoluene, 97\%, 100 g, Sigma-Aldrich Co., USA) was opened in a fume hood to avoid contamination and allowed to stand for over $1 \mathrm{~h}$ before the experiment to eliminate the difference in humidity between the inside and outside of the bottle. The handheld DNT detector was placed near the opening of the bottle to record the change in the oscillation frequency of the QCM. The purity of the DNT powder was kept high to confirm the sensing of DNT.

To replicate a field environment, a wooden box $\left(350 \times 350 \times 300 \mathrm{~mm}^{3}\right)$ filled with sand was prepared and $10 \mathrm{~g}$ of DNT powder wrapped with a cotton cloth was buried inside the sand at a depth of $150 \mathrm{~mm}$ (Fig. 4). The diameter of the sand particle was about $3 \mathrm{~mm}$.

\section{Results and Discussion}

\subsection{Sulfonated tetrafluoroethylene test result}

The sensitivity and selectivity of the sulfonated tetrafluoroethylene were examined by exposing it to various concentrations of DNT vapor and toluene vapor. DNT vapor with a concentration ranging from 1 to $100 \mathrm{ppb}$ was produced using the standard gas generator and delivered to the functionalized QCM surface. Note that the produced DNT vapor did not contain any moisture. Since the capturing molecules could easily lose their activity in a gaseous environment, examination under a dry condition is crucial for field application. 


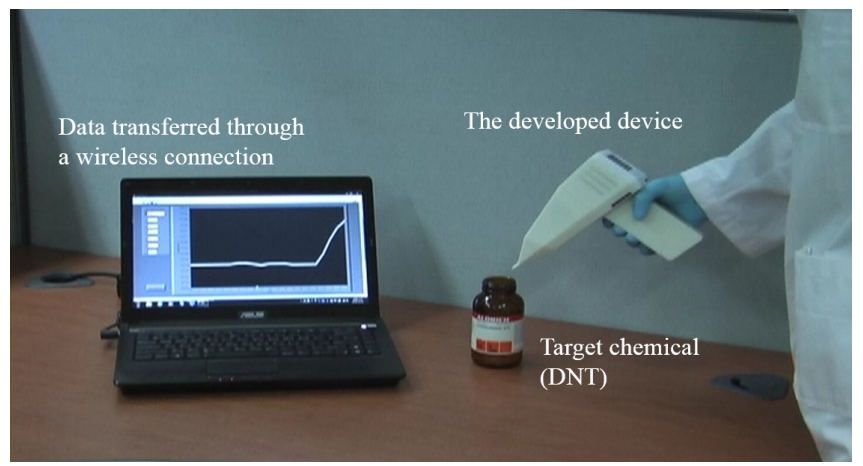

Fig. 3. (Color online) High-purity DNT bottle test. The signal of the device was monitored while the device was placed near the opening of the high-purity DNT bottle.

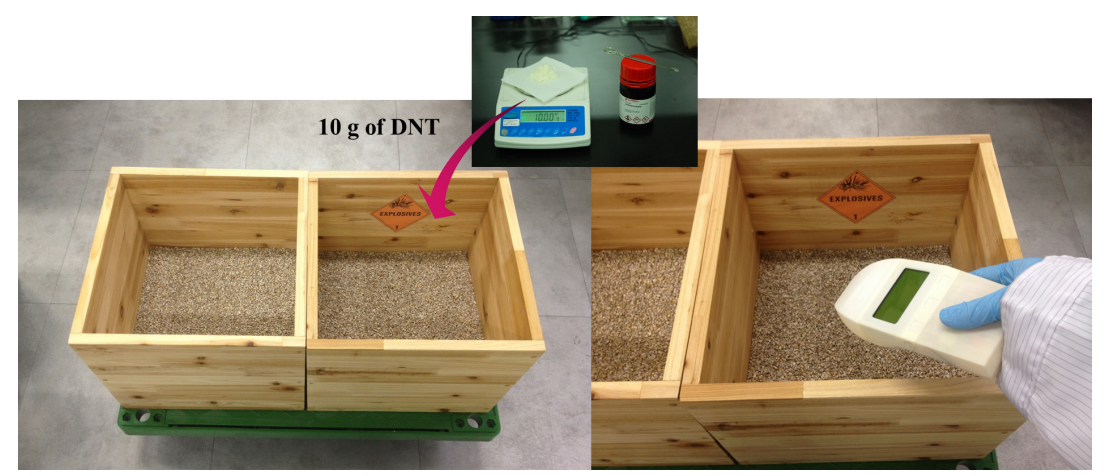

Fig. 4. (Color online) Field-reproducing setup. Two sand boxes were prepared and $10 \mathrm{~g}$ of DNT was buried in one of them. The device was placed near each box and signal changes were compared.

The QCM showed sensitivity to the DNT vapor. The frequency markedly decreased when the DNT vapor was exposed to the QCM (Fig. 5). The chemisorption of DNT on the QCM surface reduced the oscillation frequency owing to changes in mass and stress $^{(10)}$ on the surface. In contrast, the frequency was recovered by $\mathrm{N}_{2}$ purging. A change in the frequency of QCM is a very typical phenomenon with respect to the adsorption and desorption of mass on its surface. We observed a fast response within 1 min in terms of frequency change and stabilization for a particular DNT concentration. The frequency drop was recorded to be about $7.2 \mathrm{~Hz}$ in average while the QCM was exposed to $100 \mathrm{ppb}$ DNT, which could be translated to an overall sensitivity of -0.072 $\mathrm{Hz} / \mathrm{ppb}$. At a lower concentration ranging from 1 to $2 \mathrm{ppb}$, it showed a sensitivity that was 6.1 -fold higher $(-0.44 \mathrm{~Hz} / \mathrm{ppb})$ than the overall sensitivity. 


\section{Response to DNT}

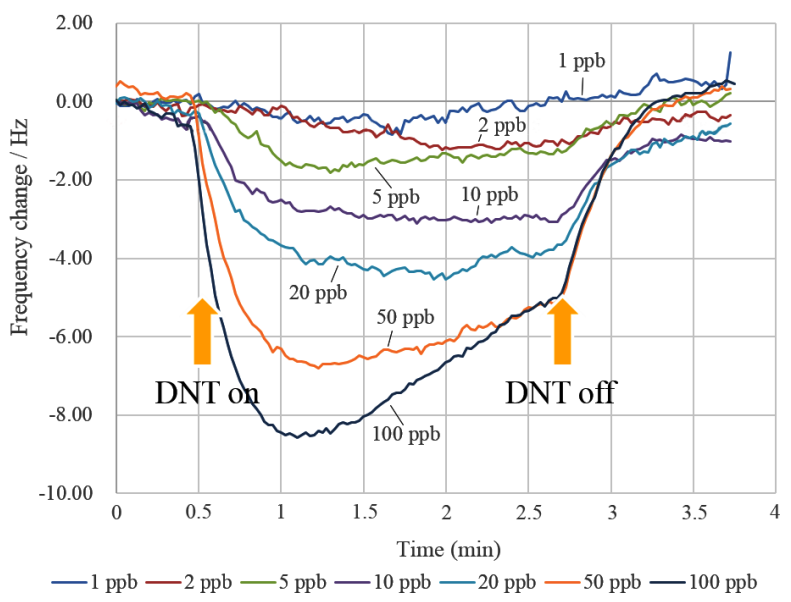

(a)
Sensitivity Characteristic Graph

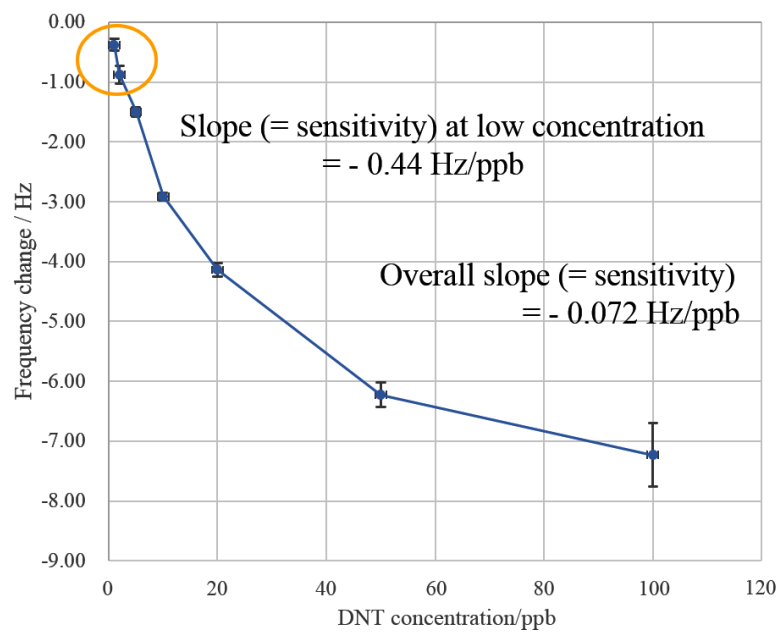

(b)

Fig. 5. (Color online) ST test result. (a) The ST-coated QCM showed a clear response to the DNT vapor. (b) ST responded more sensitively at lower DNT concentrations. The overall slope (sensitivity) was $-0.072 \mathrm{~Hz} / \mathrm{ppb}$ and the slope from 1 to $2 \mathrm{ppb}$ was $-0.44 \mathrm{~Hz} / \mathrm{ppb}$.

On the other hand, the functionalized QCM did not respond to toluene vapor (Fig. 6). Despite a high toluene concentration of $121 \mathrm{ppm}$, the QCM signal did not change in the first $5 \mathrm{~min}$ after the vapor exposure. However, after $5 \mathrm{~min}$, when the toluene concentration reached maximum $(121 \mathrm{ppm})$, the frequency started decreasing. We believed that this signal drop originated from surface condensation caused by extreme vapor pressure. After $5 \mathrm{~min}$ toluene vaporization, the vapor pressure reached the condensation threshold level. Since the condensation abruptly occurred without any sign, signal change was not observed in the first $5 \mathrm{~min}$ before the condensation. This nonspecific physical adsorption process (condensation) can occur on any surface and is not relevant to the selective interaction between ST and toluene. Even if it is, sensitivity to toluene was $-1.426 \mathrm{~Hz} / \mathrm{ppm}(=-0.0014 \mathrm{~Hz} / \mathrm{ppb})$, which is more than 50 -fold lower than that to DNT.

\subsection{DNT sensing mechanism of ST}

ST, a well-known proton exchange membrane material in fuel cell application, can be used as a selective nitroaromatic-sensing material forming the Meisenheimer complex. The Meisenheimer complex is a characteristic reaction resultant of nitroaromatic explosives and a material with a high electronegativity. It can be found in many explosive detection studies. ${ }^{(2,9,11)}$ The sulfon group $\left(\mathrm{SO}_{3}^{-}\right)$in the side chain of ST can attract nitroaromatics owing to its strong charge interactions (Fig. 7). An aromatic ring structure can be positively charged for the presence of nitrogroups, which have a high 


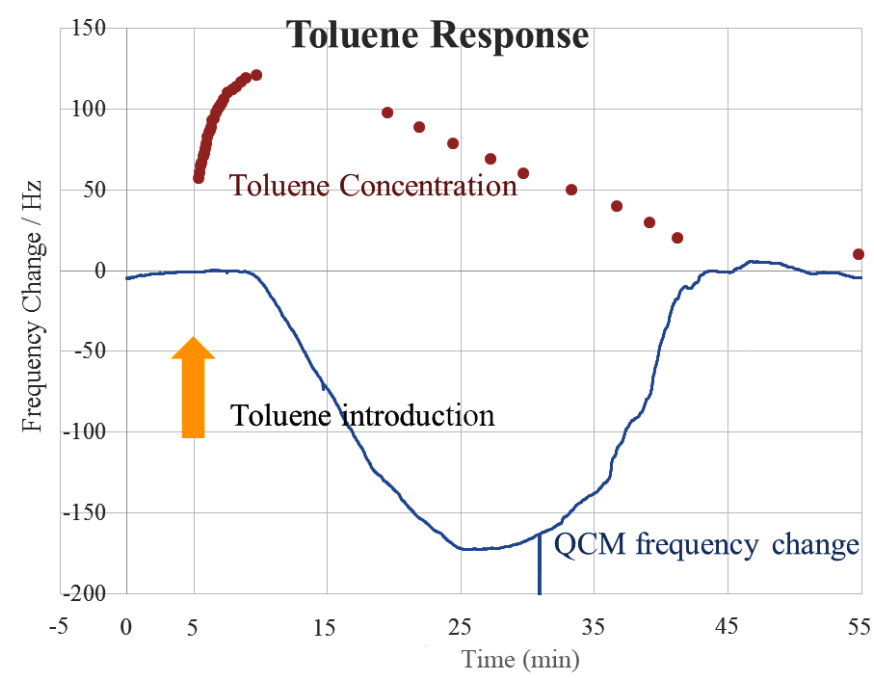

Fig. 6. (Color online) Toluene control experiment results. The QCM frequency did not change for 5 min after introducing toluene. This result shows that ST and toluene do not have chemical reactivity.

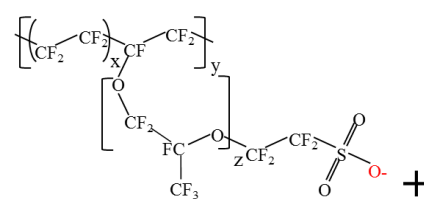

Sulfonated Tetrafluoroethylene

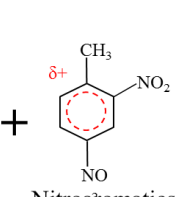

2,4-Dinitrotoluen

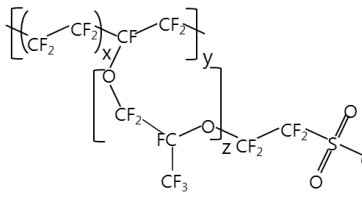

Meisenheimer

\section{Complex}

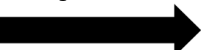<smiles>CC1(O)C=CC([N+](=O)[O-])C=C1[N+](=O)[O-]</smiles>

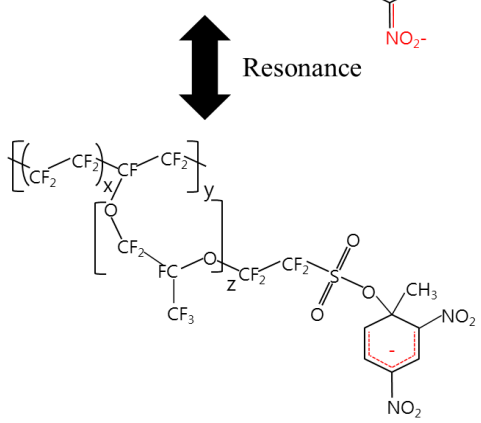

Fig. 7. (Color online) Reaction mechanism between ST and DNT. The sulfon group $\left(-\mathrm{SO}_{3}{ }^{-}\right)$in ST interacts with a positively charged aromatic ring structure in DNT and forms a stable resonance structure called the Meisenheimer complex. 
electronegativity. The positively charged aromatic ring can be attacked by an electronwithdrawing functional group, such as a sulfonated group in ST. The intermediate can be stabilized after forming a new resonance structure, the Meisenheimer complex.

Typically, it requires a wet environment, which is difficult to maintain in a field environment, to form the Meisenheimer complex. Since ionization is required prior to the reaction, maintaining a wet environment is vital to keeping sensing activity. The sensitivity of the sensor system, which relies on the Meisenheimer complex, decreases significantly or loses its functionality under a dry condition. ${ }^{(9)}$

In contrast, ST can also form the Meisenheimer complex in a dry environment. Water molecules for activating functional groups can be kept in the polymer matrix. It does not require any type of environmental chamber to keep the humid condition. Therefore, unlike other Meisenheimer-complex-forming materials, ST can maintain its functionality even under a zero humidity condition.

\subsection{Handheld device test result}

The DNT bottle test showed a highly sensitive and repeatable signal in response to a small change in DNT concentration (Fig. 8). A minimum signal drop of $55.83 \mathrm{~Hz}$ was measured every measurement. The average frequency drop was $67.04 \mathrm{~Hz}$ and the mean standard deviation of the signal drop was $13.35 \mathrm{~Hz}$. The DNT concentration at this moment was estimated as $2 \mathrm{ppb}$ using the commercial QCM that we used previously. The frequency drop of the commercial QCM setup was a maximum of $1 \mathrm{~Hz}$, which could be translated to a 1 to $2 \mathrm{ppb}$ DNT concentration on the basis of the previous ST characteristic result. When the 2 ppb DNT concentration was considered, the sensitivity of the developed device was $-33.52 \mathrm{~Hz} / \mathrm{ppb}$.

The developed device had a more than 76.2-fold higher sensitivity than the commercially available QCM setup used in this study. Since a smaller QCM could be affected more easily by the adsorbent, the sensitivity of a miniaturized sensor could be enhanced. The size of the QCM was reduced by 49.2-fold volumewise. The commercially available QCM used in this study had a 1 in $(=25.4 \mathrm{~mm})$ diameter and a 6 mill $(=1.524 \mathrm{~mm})$ thickness. The QCM developed in this work had a $10 \mathrm{~mm}$ diameter and a $0.2 \mathrm{~mm}$ thickness.

DNT bottle test result

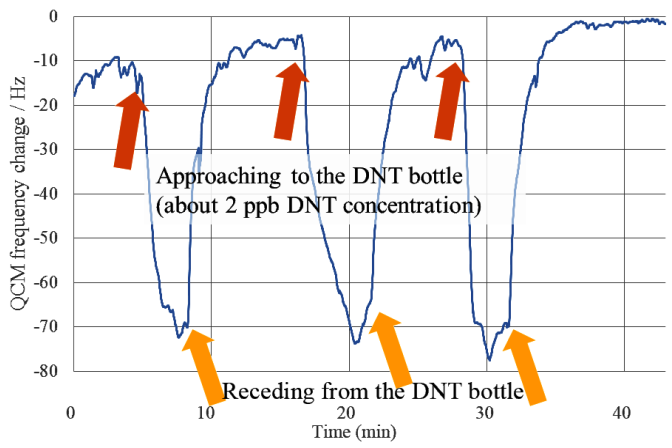

Fig. 8. (Color online) High-purity DNT bottle test result of the device. A repeatable $60 \mathrm{~Hz}$ frequency change was observed at an estimated 2 ppb DNT concentration. 
DNT buried box sensing result

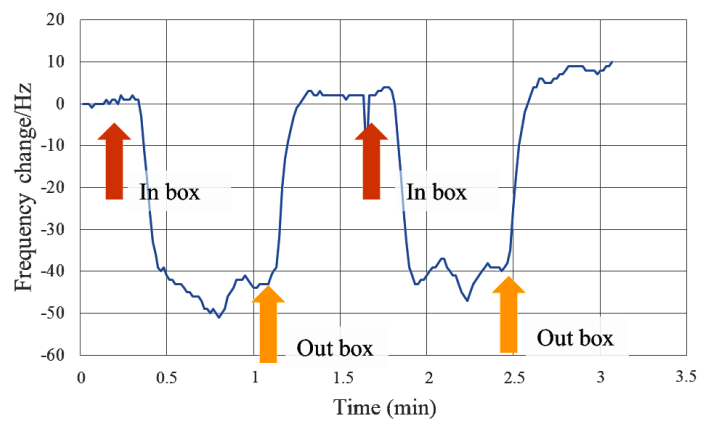

Sand box sensing result

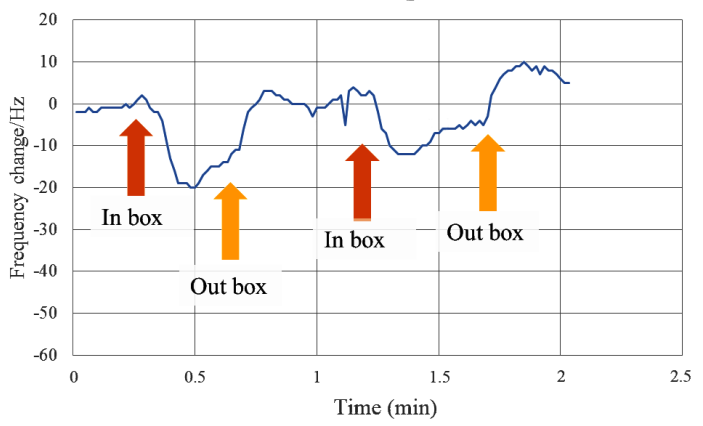

Fig. 9. (Color online) Semifield test results. The frequency change was observed to be approximately 2-fold larger in the box with DNT buried than in the box without it.

The LOD reached a value below $1.2 \mathrm{ppb}$. Since the average signal and mean standard deviation noise level were $-67.03 \mathrm{~Hz}$ (at $2 \mathrm{ppb}$ ) and $13.35 \mathrm{~Hz}$, respectively, the LOD was drawn as $1.2 \mathrm{ppb}$ by definition [LOD $=3 \times \mathrm{SD} /$ sensitivity (= slope)]. Although sensors with higher LOD performance exist, note that our device was tested in an uncontrolled environment. An uncontrolled environment may cause unpredictable noises for other gas sources, flow disturbance and local temperature change. Sensors tested in a lab environment might easily lose their functionality in a real situation. Our sensor system was sufficiently robust to ignore environmental noises.

The robustness of the sensor became more vivid in the semifield test result. The device signal dropped by $42.71 \mathrm{~Hz}$ in average, when .the device was placed near the sand with DNT (Fig. 9). On the other hand, in the control experiment (sand without DNT), the signal dropped by $11.70 \mathrm{~Hz}$ in average. An approximately 3.65-fold larger signal change was observed when the DNT was present in the sand. When the signal from the sand without DNT was assumed as a noise, the mean standard deviation noise was 7.53 Hz. The DNT sensing signal was more than 6.3 -fold $(=42.71 \mathrm{~Hz} / 7.53 \mathrm{~Hz})$ stronger than the noise, and sand particles with and without DNT were distinguishable.

\section{Conclusions}

ST showed high sensitivity and reliable DNT sensing capability. The sensing performance of ST was verified with a certified standard gas generator and a commercially available QCM. DNT vapor with a concentration ranging from 1 to 100 ppb was applied to the ST-functionalized QCM surface. The frequency response of the ST-functionalized QCM was monitored in the presence of controlled DNT vapor. The overall sensitivity ranging from 1 to $100 \mathrm{ppb}$ was $-0.072 \mathrm{~Hz} / \mathrm{ppb}$ and the sensitivity ranging from 0 to $2 \mathrm{ppb}$ was about $-0.44 \mathrm{~Hz} / \mathrm{ppb}$. In addition, ST showed excellent DNT selectivity in a control experiment with toluene. The ST-functionalized QCM did not respond to toluene at a toluene concentration of $120 \mathrm{ppm}$, which was 1200 times higher than the maximum concentration of DNT during the ST-DNT characteristic experiment mentioned above. 
More importantly, it was also observed that ST maintained its sensing activity even under a dry condition, while other sensing elements easily lose their functionality.

The sensing performance was enhanced by miniaturizing the QCM. The miniaturized quartz crystal was 49.2-fold smaller by volume than the crystal in the commercial setup. As a result, the sensing system had a 76.2-fold higher sensitivity, which was $-33.52 \mathrm{~Hz} /$ $\mathrm{ppb}$. Furthermore, the miniaturized system successively demonstrated its feasibility for field application inside a real field-mimicking setup. It showed distinguishable signal differences between a sand box with DNT buried and a sand box without it.

In conclusion, an ST-functionalized miniature QCM detector showed robust and reliable DNT sensing in an uncontrolled semifield environment. The reliability and robustness of the detector present a possibility of a new technology that can be used to search for hidden explosives in a real field environment.

\section{Acknowledgements}

This research was supported by a grant "Bio-Mimetic Robot Research Center" (UD130070ID) funded by the Defense Acquisition Program Administration and KIST Center for Intelligent Robotics funded by the Korean government (MOTIE). Fabrication and experiments were performed at the Inter-University Semiconductor Research Center, Seoul National University.

\section{References}

1 M. Burke, L. P. Vicetic, C. Caraux, K. Clarke, H. Hosman and M. Schulze: Landmine Monitor 2012 (2012) 37.

2 S. Singh: J. Hazard. Mater. 144 (2007) 15.

3 M. Cerruti, J. Jaworski, D. Raorane, C. Zueger, J. Varadarajan, C. Carraro, S.-W. Lee, R. Maboudian and A. Majumdar: Anal. Chem. 81 (2009) 4192.

4 K. Nagatomo, T. Kawaguchi, N. Miura, K. Toko and K. Matsumoto: Talanta 79 (2009) 1142.

5 E. J. Houser, T. E. Mlsna, V. K. Nguyen, R. Chung, R. L. Mowery and R. A. McGill: Talanta 54 (2001) 469.

6 H. Wang, X. Xu, C. Lee, C. Johnson, K. Sohlberg and H.-F. Ji: J. Phys. Chem. C 116 (2012) 4442.

7 L. A. Pinnaduwage, T. Thundat, J. E. Hawk, D.L. Hedden, R. Britt, E. J. Houser, S. Stepnowski, R. A. McGill and D. Bubb: Sens. Actuator B 99 (2004) 223.

8 P. T. Charles, A. A. Adams, P. B. Howell, S. A. Trammell, J. R. Deschamps and A. W. Kusterbeck: Sensors 10 (2010) 876.

9 Y. Engel, R. Elnathan, A. Pevzner, G. Davidi, E. Flaxer and F. Patolsky: Angew. Chem. 49 (2010) 6830.

10 L. Tan, Q. Xie, X. Jia, M. Guo, Y. Zhang, H. Tang and S. Yao: Biosens. Bioelectron. 24 (2009) 1603.

11 J. L. Pablos, M. Trigo-Lopez, F. Serna, F. C. Garcia and J. M. Garcia: RSC Adv. 4 (2014) 25562. 